\title{
Study of the Energy of the Free Oscillations of the Earth ${ }^{1}$
}

\author{
ROBERT L. KOVACH \\ Department of Geophysics, Stanford University, Stanford, California \\ Don L. Anderson \\ Seismological Laboratory, California Institute of Technology, Pasadena
}

\begin{abstract}
The energies of the radial, torsional, and spheroidal free oscillations for a Gutenberg model earth were studied. Each mode of oscillation has a characteristic radial distribution of elastic and kinetic energy that fixes the parts of the earth that contribute most heavily in determining a particular resonant frequency. An examination of the partitioning of energy among compressional, shear, and gravitational energy as a function of mode number and depth immediately explains the persistence of the purely radial mode compared with the other normal modes of the earth. Only the first few spheroidal modes are sensitive to the density of the inner core; they are particularly sensitive to the density of the outer part of the core. The low-order spheroidal modes also exhibit a rapid rise of potential energy near the base of the mantle; this rise will permit improved estimates of the velocity to be obtained in this region, which is difficult to examine with body waves. The tabulated results allow estimates to be made of the previously neglected energy contained in the free oscillations excited by large earthquakes. An estimate of the energy in the low-order spheroidal oscillations excited by the great Alaskan shock suggests a value of $10^{23}$ ergs over the period range from 450 to $830 \mathrm{sec}$, implying that the energy density increases toward high frequencies if the total energy in the earthquake was of the order of $10^{24}-10^{25}$ ergs.
\end{abstract}

\section{INTRODUCTION}

The energy of an earthquake is a fundamental quantity that bears directly on the problem of the energy budget of the earth. It is well known, however, that the methods used to measure the seismic energy emitted by the source are far from satisfactory. Estimates of earthquake energy are usually made by means of an empirical relationship between magnitude and energy, but it is not clear what part of the frequency spectrum is being considered. Calibration of the earthquake magnitude scale, using nuclear sources of known energy, are refining this relationship, but this method requires assumptions on the efficiency of generating seismic energy and is complicated by the possibility that pre-existing tectonic strain is released by the explosion.

Even the most direct estimates of earthquake energy have utilized only short-period surface waves and free oscillations. One purpose of the present paper is to provide tables that allow

\footnotetext{
${ }^{1}$ Contribution 1379, Division of Geological Sciences, California Institute of Technology.
}

estimates to be made of the energy contained in long-period surface waves and free oscillations. This energy has been neglected in previous work.

This study is one step in the program of improving estimates of the energy involved in earthquakes. The increased density of seismometers provided by the U. S. Coast and Geodetic Survey worldwide network combined with increasing sophistication in measuring and computing radiation patterns are other important improvements that will help take into account all the energy radiated by a seismic source. Recent contributions to this end have been made by Harkrider and Anderson [1966], Haskell [1966], and Harkrider [1966]. Wu [1966] has made the most complete study to date of the energy budget of earthquakes.

It is possible to calibrate the intensity of large earthquakes that have excited the free oscillations of the earth by measuring the energies contained in specific modes of oscillation. The surface displacement for a given frequency at a point on the earth's surface specifies the total kinetic energy in that mode of oscillation, and the variation of total energy with mode 
number determines the energy spectrum of the earthquake.

The energy of surface waves and free oscillations has been treated theoretically by Jeffreys [1923], Jobert and Jobert [1953], Pekeris and Jarosch [1958], Stoneley [1958], Anderson [1964], and Takeuchi et al. [1964]. A preliminary study of the energy in the low-order torsional modes was made by MacDonald and Ness [1961].

We shall derive the formal expressions for computing the energies in any mode of oscillation for the torsional and spheroidal oscillations and present numerical results for a realistic earth model. An estimate is made of the total energy in several of the spheroidal modes excited by the great Alaskan earthquake of 1964 .

The results in this paper can be used to convert observed surface wave and free oscillation spectra to total energy in the modes of frequency band being measured. In addition, the partitioning of energy and the variation of energy with depth for various modes are presented. In a later paper we will use this information to revise the elastic, density, and anelastic structure of the earth, following Anderson [1964] and Anderson and Archambeau [1964]. The perturbation parameters required for such a study are simple ratios of the energy integrals computed for the present paper; tables of these parameters will be published separately.

\section{Symbols}

a, unit vector in radial or angular direction.

$\mathbf{B}_{l^{m}}, \mathbf{C}_{l^{m}}, \mathbf{P}_{l^{m}}$, vector spherical harmonics of angular order $l, m$.

$\Delta$, dilatation.

$\epsilon_{i}$, elastic strain tensor.

$g$, gravitational acceleration.

$G$, gravitational constant.

$l$, integer denoting surface harmonic dependence on polar angle.

$\lambda$, Lamé constant.

$\mu$, rigidity.

$m$, integer denoting azimuthal dependence.

$n$, number of radial nodal surfaces.

$P_{\imath}{ }^{m}(\theta, \phi)$, surface spherical harmonic.

$P(r)$, radial factor in the perturbation of the gravitational potential.
$\Psi_{0}$, unperturbed gravitational potential.

$\Psi$, perturbation in the gravitational potential.

q, complex amplitude of surface displacement.

$\rho$, density.

$\tau_{i i}$, elastic stress tensor.

$\mathfrak{u}_{a}$, spheroidal oscillation surface displacement.

$\mathfrak{u}_{t}$, torsional oscillation surface displacement.

$u$, vertical component of surface displacement for spheroidal oscillations.

${ }_{n} U_{\mathbf{l}}(r)$, radial function in the vertical component of displacement for spheroidal oscillations.

$v$, horizontal component of surface displacement.

${ }_{n} V_{l}(r)$, radial function in horizontal component of displacement.

$w$, horizontal component of surface displacement.

${ }_{n} W_{l}(r)$, radial function in the torsional oscillations.

$\omega$, angular frequency.

$X(r)$, radial factor in the dilatation.

\section{Basic Theory}

The spheroidal and torsional motions of a vibrating sphere can be expressed in terms of vector spherical harmonics $\mathrm{B}_{l}^{m}(\theta, \phi), \mathrm{C}_{l}^{m}(\theta, \phi)$, $\mathbf{P}_{\imath}{ }^{m}(\theta, \phi)$

$$
\begin{aligned}
& \mathbf{u}^{\prime}= \sum_{l=0}^{\infty} \sum_{m=-l}^{l} \sqrt{l(l+1)} \mathbf{C}_{l}{ }^{m}(\theta, \phi) \\
& \cdot D_{l}{ }^{m}{ }_{n} W_{l}(r) e^{i \omega t} \\
& \mathbf{u}^{5}= \sum_{l=0}^{\infty} \sum_{m=-l}^{l}\left(\mathbf{P}_{l}{ }^{m}(\theta, \phi) A_{l}{ }^{m}{ }_{n} U_{l}(r)\right. \\
&\left.+\sqrt{l(l+1)} \mathbf{B}_{l}{ }^{m}(\theta, \phi) A_{l}{ }^{m}{ }_{n} V_{l}(r)\right) e^{i \omega t}
\end{aligned}
$$

where $A_{l}{ }^{m}$ and $D_{l}{ }^{m}$ are weighting factors for each value of $m$ and where

$$
\begin{gathered}
\mathbf{P}_{l}^{m}(\theta, \phi)=\mathbf{a}_{r} e^{i m \phi} P_{l}^{m}(\cos \theta) \\
\sqrt{l(l+1,} \mathbf{C}_{l}^{m}(\theta, \phi)=\mathbf{a}_{\theta} \frac{i m}{\sin \theta} \\
\cdot e^{i m \phi} P_{l}^{m}(\cos \theta)-\mathbf{a}_{\phi} e^{i m \phi} \frac{\partial P_{l}^{m}(\cos \theta)}{\partial \theta}
\end{gathered}
$$


$\sqrt{l(l+1,} \mathbf{B}_{l}^{m}(\theta, \phi)=\mathbf{a}_{\theta} e^{i m \phi} \frac{\partial P_{l}^{m}(\cos \theta)}{\partial \theta}$

$$
+\mathbf{a}_{\phi} \frac{i m}{\sin \theta} e^{i m \phi} P_{l}^{m}(\cos \theta)
$$

[Morse and Feshbach, 1953]. For example,

$$
\begin{aligned}
& \mathbf{q}_{r}=\sum_{l=0}^{\infty} \sum_{m=-l}^{l} \mathbf{a}_{r} A_{l}{ }^{m} P_{l}^{m}(\cos \theta) e^{i m \varphi}, U_{l}(r) \\
& \mathbf{q}_{\theta}=\sum_{l=0}^{\infty} \sum_{m=-l}^{l} \mathbf{a}_{\theta} A_{l}{ }^{m} \frac{\partial P_{l}^{m}(\cos \theta)}{\partial \theta} e^{i m r}{ }_{n} V_{l}(r) \\
& \mathbf{q}_{\phi}=\sum_{l=0}^{\infty} \sum_{m=-l}^{l} \mathbf{a}_{i} A_{l}{ }^{m} \frac{i m P_{l}^{m}(\cos \theta)}{\sin \theta} e^{i m{ }_{n} V_{l}(r)}
\end{aligned}
$$

define the complex amplitudes of the three orthogonal components of displacement for the spheroidal oscillations, taking the time variation of the displacement to be of the form $\mathbf{u}=q e^{i \omega t}$. ${ }_{n} U_{l}(r)$ and ${ }_{n} V_{l}(r)$, the radial factors in the vertical and horizontal components of displacement, depend on the distribution of elastic parameters with depth.

The kinetic energy averaged over a cycle for an oscillation of frequency $\omega$ is given by the expression

$$
\frac{\omega^{2}}{4} \iiint \rho(r) \mathbf{q}_{i} \mathbf{q}_{i}{ }^{*} d V
$$

where $q_{i}{ }^{*}$ is the complex conjugate of $q_{i}$. For the spheroidal oscillations the kinetic energy associated with an oscillation of order $l$ and degree $m$ is

$$
\begin{aligned}
\left(A_{l}{ }^{m}\right)^{2} & \frac{\omega^{2} \pi / \epsilon_{m}(l+m) !}{(2 l+1)(l-m) !} \int_{0}^{a} \rho(r) r^{2} \\
& \cdot\left({ }_{n} U_{l}{ }^{2}(r)+l(l+1){ }_{n} V_{l}{ }^{2}(r)\right) d r
\end{aligned}
$$

using the orthogonality relations

$$
\begin{aligned}
\int \mathbf{C}_{l}{ }^{m} \mathbf{C}_{l}{ }^{m^{*}} d S & =\int \mathbf{P}_{l}{ }^{m} \mathbf{P}_{l}{ }^{m^{*}} d S \\
& =\int \mathbf{B}_{l}{ }^{m} \mathbf{B}_{l}{ }^{m^{*}} d S=\frac{4 \pi / \epsilon_{m}(l+m) !}{(2 l+1)(l-m) !}
\end{aligned}
$$

where $\epsilon_{m}=1$, if $m=0$, and $\epsilon_{m}=2$, if $m \neq 0$. Similarly for the torsional oscillations the kinetic energy becomes

$$
\begin{aligned}
& \left(D_{l}^{m}\right)^{2} \frac{l(l+1) \omega^{2} \pi / \epsilon_{m}(l+m) !}{(2 l+1)(l-m) !} \\
& \cdot \int_{0}^{r} \rho(r){ }_{n} W_{l}{ }^{2}(r) r^{2} d r
\end{aligned}
$$

The elastic or potential energy averaged over a cycle is

$$
\frac{1}{4} \iiint \tau_{i j} e_{i ;} d V
$$

where $\tau_{i}$ are the stresses on the spherical surfaces and $e_{i j}$ are the corresponding strains. Writing the stress-strain relations in spherical coordinates [Love, 1927] and making use of the relations

$$
\begin{aligned}
د & =X(r) P_{l}^{m}(\theta, \phi) \\
\mathrm{X}(r) & =\frac{\partial U_{l}(r)}{\partial r}+\frac{2}{r} U_{l}(r)-\frac{l(l+1) V_{l}(r)}{r}
\end{aligned}
$$

and the partial differential equation

$$
\begin{aligned}
& \frac{1}{\sin ^{2} \theta} \frac{\partial^{2} P_{l}^{m}(\theta, \phi)}{\partial \phi^{2}}+\frac{1}{\sin \theta} \frac{\partial}{\partial \theta} \\
& \quad\left(\sin \theta \frac{\partial P_{l}^{\prime \prime}(\theta, \phi)}{\partial \theta}\right)=-l(l+1) P_{l}^{\prime \prime}(\theta, \phi)
\end{aligned}
$$

[Hoskins, 1920, p. 10], the elastic energy for the spheroidal oscillations can be put in the form

$$
\begin{aligned}
& \frac{\left(A_{l}{ }^{m}\right)^{2} \pi / \epsilon_{m}(l+m) !}{(2 l+1)(l-m) !} \int_{n}^{r}\{(\lambda+2 \mu) \\
& \cdot\left(r \frac{\partial U_{l}}{\partial r}+2 U_{l}-l(l+1) V_{l}\right)^{2} \\
& +\mu l(l+1)\left(r \frac{\partial V_{l}}{\partial r}-V_{l}+V_{l}\right)^{2} \\
& -4 \mu U_{l}{ }^{2}-2 l(l+1) \mu V_{l}{ }^{2}+4 l(l+1) V_{l} \\
& \left.\cdot\left(\mu U_{l}+\mu r \frac{\partial U_{l}}{\partial r}\right)-\operatorname{s\mu r} U_{l} \frac{\partial U_{l}}{\partial r}\right\} d r
\end{aligned}
$$

Similarly, the elastic or potential energy for the torsional oscillations is

$$
\begin{gathered}
\frac{\left.\left(D_{l}\right)^{2}\right)^{2} \pi / \epsilon_{m}(l+m) ! l(l+1)}{(2 l+1)(l-m) !} \int_{0}^{r} \mu(r)\left\{\left(\frac{\partial W_{l}(r)}{\partial r}\right.\right. \\
\left.\left.-\frac{W_{l}(r)}{r}\right)^{2}+\left(l^{2}+l-2\right) \frac{W_{l}{ }^{2}(r)}{r^{2}}\right\} r^{2} d r
\end{gathered}
$$

which agrees with the expression given by $\mathrm{Mac}$ Donald and Ness [1961].

Because the spheroidal oscillations perturb the gravitational field, the gravitational energy must also be computed. The strains caused by gravitational perturbations may be treated as being due to three sets of bodily forces [Hos- 
kins, 1920]: (1) forces due to the perturbation of the gravitational potential, including the disturbing force and the change in gravitational force caused by the changed configuration of the attracting mass; (2) forces having a potential $u d \Psi_{0} / d r$ where $u$ is the radial displacement and $\Psi_{0}$ is the unperturbed gravitational potential; and (3) the increment of force due to the change $-\rho \Delta$ in the density of the attracted mass.

We may, therefore, write the gravitational force in the radial direction as

$$
\rho \frac{d \Psi_{0}}{d r} \Delta-\rho \frac{\partial}{\partial r}\left(\Psi+u \frac{d \Psi_{0}}{d r}\right)
$$

where $\Psi$ is the perturbation in the gravitational potential. Similarly, the forces in the $\theta$ and $\phi$ directions are

$$
\begin{aligned}
& \rho\left\{-\frac{1}{r} \frac{\partial}{\partial \theta}\left(\Psi+\mathrm{u} \frac{d \Psi_{n}}{d r}\right)\right\} \\
& \rho\left\{-\frac{1}{r \sin \theta} \frac{\partial}{\partial \phi}\left(\Psi+\mathrm{u} \frac{d \Psi_{0}}{d r}\right)\right\}
\end{aligned}
$$

Then the gravitational energy can be written as

$$
\begin{aligned}
\iiint\left(\left\{\rho \frac{d \Psi_{0}}{d r}\right.\right. & \Delta-\rho \frac{\partial \Psi}{\partial r} \\
& \left.-\rho u \frac{d^{2} \Psi_{0}}{d r^{2}}-\rho \frac{d \Psi_{0}}{d r} \frac{d u}{d r}\right\} u \\
& +\left\{-\frac{\rho}{r} \frac{\partial \Psi}{\partial \theta}-\frac{\rho}{r}\left(\frac{d \Psi_{0}}{d r} \frac{\partial u}{\partial \theta}\right)\right\} v \\
& \left.-\frac{\rho}{r \sin \theta}\left\{\frac{\partial \Psi}{\partial \phi}+\frac{d \Psi_{0}}{d r} \frac{\partial u}{\partial \phi}\right\} w\right) d V
\end{aligned}
$$

Making the substitutions

$$
\begin{aligned}
\Delta & =X(r) P_{l}{ }^{m}(\theta, \phi) \\
\Psi & =P(r) P_{l}{ }^{m}(\theta, \phi) \\
\frac{d \Psi_{0}}{d r} & =-g \\
\frac{d^{2} \Psi_{0}}{d r^{2}} & =-4 \pi G \rho+\frac{2 g}{r} \\
X(r) & =\frac{\partial U_{l}(r)}{\partial r}+\frac{2}{r} U_{l}(r)-\frac{l(l+1) V_{l}(r)}{r} \\
u & =A_{l}{ }^{m}{ }_{n} U_{l}(r) P_{l}{ }^{m}(\theta, \varphi) \\
v & =A_{l}{ }^{m}{ }_{n} V_{l}(r) \frac{\partial P_{l}{ }^{m}(\theta, \varphi)}{\partial \theta}
\end{aligned}
$$

$$
w=A_{l}{ }^{m} \frac{{ }_{n} V_{l}(r)}{\sin \theta} \frac{\partial P_{l}{ }^{m}(\theta, \varphi)}{\partial \varphi}
$$

and after some manipulation, the gravitational energy averaged over a cycle becomes

$$
\begin{aligned}
& \frac{\left(A_{l}{ }^{m}\right)^{2} \pi / \epsilon_{m}(l+m) !}{(2 l+1)(l-m) !} \int_{0}^{r}\left\{-4 \rho g U_{l}^{2}(r) r\right. \\
& \quad-\rho \frac{d P(r)}{d r} U_{l}(r) r^{2}+4 \pi G \rho^{2} U_{l}^{2}(r) r^{2} \\
& \quad+2 \rho g U_{l}(r) V_{l}(r) r l(l+1) \\
& \left.\quad-l(l+1) \rho P(r) V_{l}(r) r\right\} d r
\end{aligned}
$$

\section{Accuracy of the Results}

The accuracy of the numerical integrations for the kinetic energy were checked against exact calculations for a homogeneous gravitating sphere. For the purely radial mode $l=0$, so that the kinetic energy of a homogeneous sphere can be written as

$$
\omega^{2} \pi \bar{\rho} \int_{0}^{a} r^{2} U_{0}^{2}(r) d r
$$

The displacement factor for radial oscillations is

$$
U_{0}(r)=\frac{\sin k r}{k^{2} r^{2}}-\frac{\cos k r}{k r}
$$

where

$$
k=\bar{\rho}\left(\omega^{2}+4 A\right) /(\lambda+2 \mu)
$$

and

$$
4 A=(16 \pi / 3) G \bar{\rho}
$$

[Pekeris and Jarosch, 1958].

The above energy integral is easily integrated to

$$
\frac{\omega^{2} \pi \bar{\rho}}{k^{3}}\left\{\frac{k a}{2}-\frac{\sin ^{2} k a}{k a}+\frac{\sin 2 k a}{4}\right\}
$$

This expression was evaluated for model $\beta$, which is a homogeneous solid with properties equal to the average over the globe of Bullen's model B [Alterman et al., 1959], and the results were compared with numerical integration results. The results agree to $3 \%$ with the modified trapezoidal integration scheme and step size used in the evaluation of the energy integrals. In the numerical calculations that follow results are presented for a GutenbergBullen A model (Table 1). 
TABLE 1. Gutenberg-Bullen A Layer Parameters

\begin{tabular}{rrrr} 
Depth, km & $\alpha, \mathrm{km} / \mathrm{sec}$ & $\beta, \mathrm{km} / \mathrm{sec}$ & $\rho, \mathrm{g} / \mathrm{cm}^{3}$ \\
\hline 0 & 6.14 & 3.55 & 2.75 \\
19 & 6.14 & 3.55 & 2.75 \\
19 & 6.58 & 3.80 & 2.90 \\
38 & 6.58 & 3.80 & 2.90 \\
38 & 8.20 & 4.65 & 3.32 \\
50 & 8.20 & 4.65 & 3.32 \\
55 & 8.17 & 4.62 & 3.34 \\
65 & 8.14 & 4.57 & 3.35 \\
85 & 8.07 & 4.46 & 3.37 \\
95 & 8.02 & 4.41 & 3.38 \\
110 & 7.95 & 4.37 & 3.39 \\
130 & 7.85 & 4.35 & 3.40 \\
150 & 7.86 & 4.35 & 3.42 \\
170 & 7.92 & 4.36 & 3.44 \\
190 & 7.98 & 4.38 & 3.46 \\
210 & 8.08 & 4.41 & 3.48 \\
230 & 8.17 & 4.44 & 3.49 \\
250 & 8.27 & 4.49 & 3.51 \\
270 & 8.36 & 4.53 & 3.53 \\
290 & 8.46 & 4.58 & 3.54 \\
310 & 8.56 & 4.64 & 3.56 \\
330 & 8.66 & 4.70 & 3.58 \\
350 & 8.76 & 4.76 & 3.60 \\
370 & 8.86 & 4.84 & 3.61 \\
390 & 8.96 & 4.92 & 3.63 \\
410 & 9.06 & 4.98 & 3.64 \\
500 & 9.60 & 5.30 & 3.89 \\
600 & 10.10 & 5.60 & 4.13 \\
700 & 10.50 & 5.90 & 4.31 \\
800 & 10.90 & 6.15 & 4.49 \\
900 & 11.30 & 6.30 & 4.59 \\
1000 & 11.40 & 6.35 & 4.68 \\
1200 & 11.80 & 6.50 & 4.80 \\
1400 & 12.05 & 6.60 & 4.92 \\
1600 & 12.30 & 6.75 & 5.02 \\
1800 & 12.55 & 6.85 & 5.14 \\
2000 & 12.80 & 6.95 & 5.24 \\
2200 & 13.00 & 7.00 & 5.34 \\
2400 & 13.20 & 7.10 & 5.44 \\
2800 & 13.70 & 7.25 & 5.64 \\
2898 & 13.65 & 7.20 & 5.69 \\
2898 & 8.10 & & 9.40 \\
3000 & 8.23 & & 9.55 \\
3500 & 8.90 & & 10.15 \\
4000 & 9.50 & & 10.70 \\
4500 & 9.97 & & 11.20 \\
4982 & 10.44 & & 11.50 \\
5121 & 9.47 & & 14.20 \\
5121 & 11.16 & & 16.80 \\
6371 & 11.31 & & 17.20 \\
\hline & & & \\
\hline & & & \\
\hline & & & \\
\hline
\end{tabular}

\section{Numerical Results}

The total elastic or kinetic energy as a function of the angular order number for the fundamental mode torsional oscillations is shown in
Figure 1 and Table 2. The energies, in ergs, are normalized by setting $m=0$ and the radial factor of the surface displacement, ${ }_{n} W_{l}(r)$, equal to $1 \mathrm{~cm}$ at the earth's surface.

The integers $l$ and $m$ specify the surface pattern of displacement for a particular mode of oscillation. There are $2 m$ nodal lines associated with the angular coordinate $\phi$, and $l-|m|$ nodal lines associated with the angular coordinate $\theta$. For computation of the elastic energy $m$ can be set equal to zero, since the energy is simply related to the azimuthal order number $m$. The energy for other values of $m$ is obtained by multiplying by the expression $(l+m) ! /$ $(l-m)$ !

Figure 1 shows that in progressing from ${ }_{0} T_{:}$ to $T_{05}$ the kinetic or elastic energy increases by about 3 orders of magnitude for a fixed surface displacement of $1 \mathrm{~cm}$, which expresses, primarily, the direct relation of energy to the square of the angular frequency. For an equivalent order number the energy in the first higher mode is about 2 orders of magnitude greater than the energy in the fundamental mode (Table 3 ). Since the behavior of the torsional oscillations is relatively simple, we shall restrict the remainder of our discussion to the more complicated spheroidal modes.

The radial displacement factors for the purely radial mode and the first two spheroidal modes are shown in Figure 2. The radial mode involves simple contraction and expansion; the vertical displacement function increases slightly to a depth of $500 \mathrm{~km}$ and then decreases uniformly to zero at the center of the earth. The spheroidal modes involve both vertical and horizontal motion. For the low-order modes both the vertical and the horizontal components of motion are largest at the core-mantle boundary. $U$ and $V$ have the same sign at the free surface only for $l=2$; for $l \geq 3$ the horizontal and vertical displacement factors have opposite signs.

The variation of energy with depth indicates to what extent the various regions of the earth contribute to the properties of a resonance peak. Figure 3 gives the energy density as a function of depth for the radial mode. The total elastic energy, which is proportional to $(\lambda+2 \mu)(r$ $d U / d r+2 U)^{2}$ is fairly uniform throughout the mantle and core.

To a depth of $1600 \mathrm{~km}$ the total elastic en- 


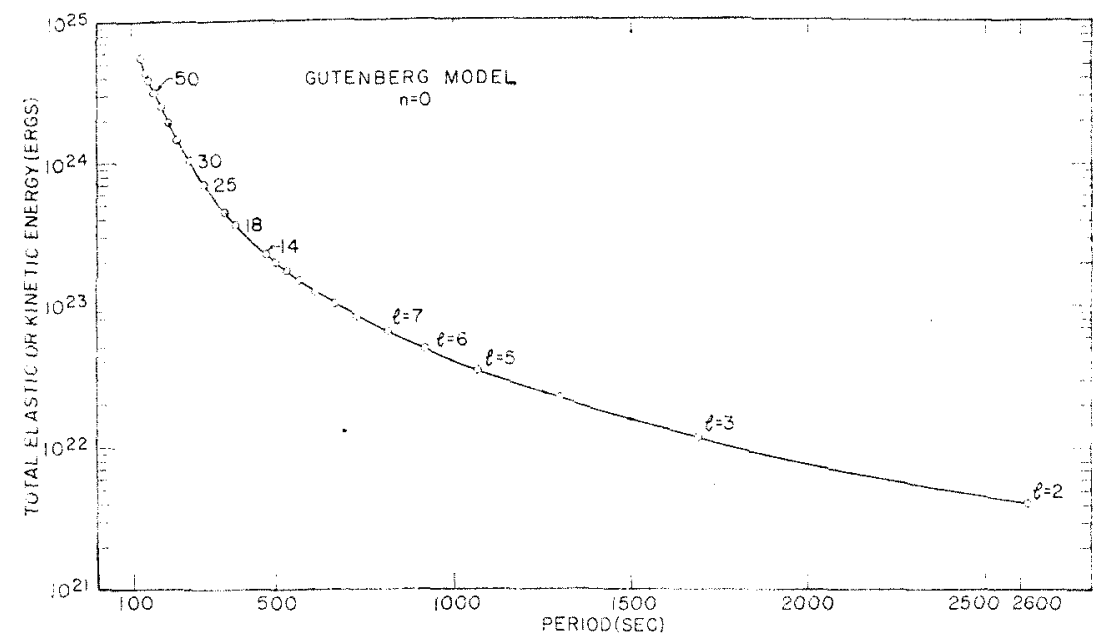

Fig. 1. Total elastic or kinetic energy versus period for fundamental mode torsional oscillations. Normalized to a $1-\mathrm{cm}$ surface displacement.

ergy per unit shell is somewhat less than the kinetic energy. In the core the rigidity vanishes, and all the elastic energy is contributed by the term containing $\lambda$, which within the fluid core is equal to the bulk modulus. The energy curve shows that the elastic parameters of the deep

TABLE 2. Energies for ${ }_{0} T_{l}$

\begin{tabular}{rrr}
\hline & & $\begin{array}{c}\text { Kinetic Energy, } \\
\text { ergs }\end{array}$ \\
\hline 2 & 2616 & $0.408 \times 10^{22}$ \\
3 & 1693 & $1.19 \times 10^{22}$ \\
4 & 1297 & $2.25 \times 10^{22}$ \\
5 & 1070 & $3.51 \times 10^{22}$ \\
6 & 921.2 & $4.94 \times 10^{22}$ \\
7 & 814.4 & $6.55 \times 10^{22}$ \\
8 & 733.2 & $8.32 \times 10^{22}$ \\
9 & 669.0 & $10.3 \times 10^{22}$ \\
10 & 616.7 & $12.4 \times 10^{22}$ \\
11 & 572.9 & $14.7 \times 10^{22}$ \\
12 & 535.8 & $17.2 \times 10^{22}$ \\
13 & 503.7 & $19.9 \times 10^{22}$ \\
14 & 475.6 & $22.8 \times 10^{22}$ \\
18 & 390.9 & $36.7 \times 10^{22}$ \\
20 & 359.6 & $45.1 \times 10^{22}$ \\
25 & 300.5 & $70.8 \times 10^{22}$ \\
30 & 258.5 & $104 \times 10^{22}$ \\
35 & 227.0 & $144 \times 10^{22}$ \\
40 & 202.3 & $192 \times 10^{22}$ \\
45 & 182.6 & $249 \times 10^{22}$ \\
50 & 166.3 & $314 \times 10^{22}$ \\
55 & 152.8 & $387 \times 10^{22}$ \\
60 & 141.2 & $468 \times 10^{22}$ \\
65 & 131.3 & $558 \times 10^{22}$ \\
& & \\
\hline
\end{tabular}

mantle and outer core and the density of the upper mantle contribute most heavily to the radial mode of oscillation.

The kinetic energy per unit thick shell reaches a maximum at a depth of about $800 \mathrm{~km}$ and then decreases uniformly to the core boundary. At the core boundary the kinetic energy increases sharply because of the density jump into the core. The small discontinuity at a depth of $5120 \mathrm{~km}$ is due to the density effect of the inner core.

The $\lambda$ and $\mu$ and bulk modulus ( $k$ ) components of the elastic energy are also shown in Figure 3. Within the mantle the elastic energy contributed by the bulk modulus term is a factor of about 1.5 to 2.5 times larger than that contributed from the rigidity term, which shows that the mode $S_{0}$ is primarily a compressional mode. In the core, of course, all the elastic energy is compressional. Since losses in com-

TABLE 3. Energies for ${ }_{1} T_{l}$

\begin{tabular}{ccc} 
Order, $l$ & $T$, sec & $\begin{array}{c}\text { Kinetic Energy, } \\
\text { ergs }\end{array}$ \\
\hline 2 & 753.6 & $0.041 \times 10^{24}$ \\
3 & 691.1 & $0.073 \times 10^{24}$ \\
4 & 627.2 & $0.120 \times 10^{24}$ \\
5 & 568.0 & $0.183 \times 10^{24}$ \\
10 & 379.4 & $0.644 \times 10^{24}$ \\
14 & 305.7 & $1.22 \times 10^{24}$ \\
17 & 268.4 & $1.80 \times 10^{24}$
\end{tabular}




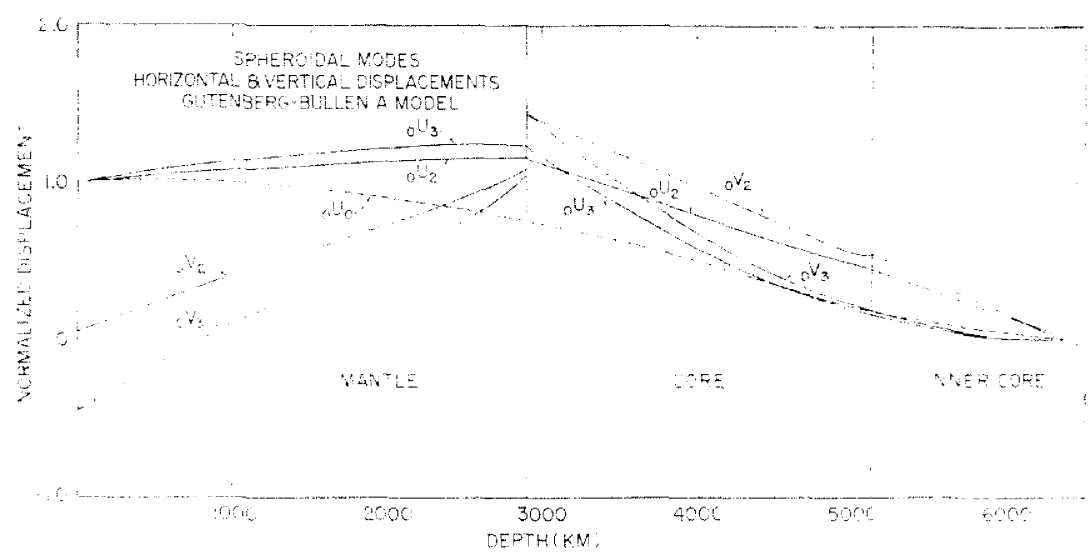

Fig. 2. Displacements versus depth for the radial mode and the first two low-order spheroidal modes.

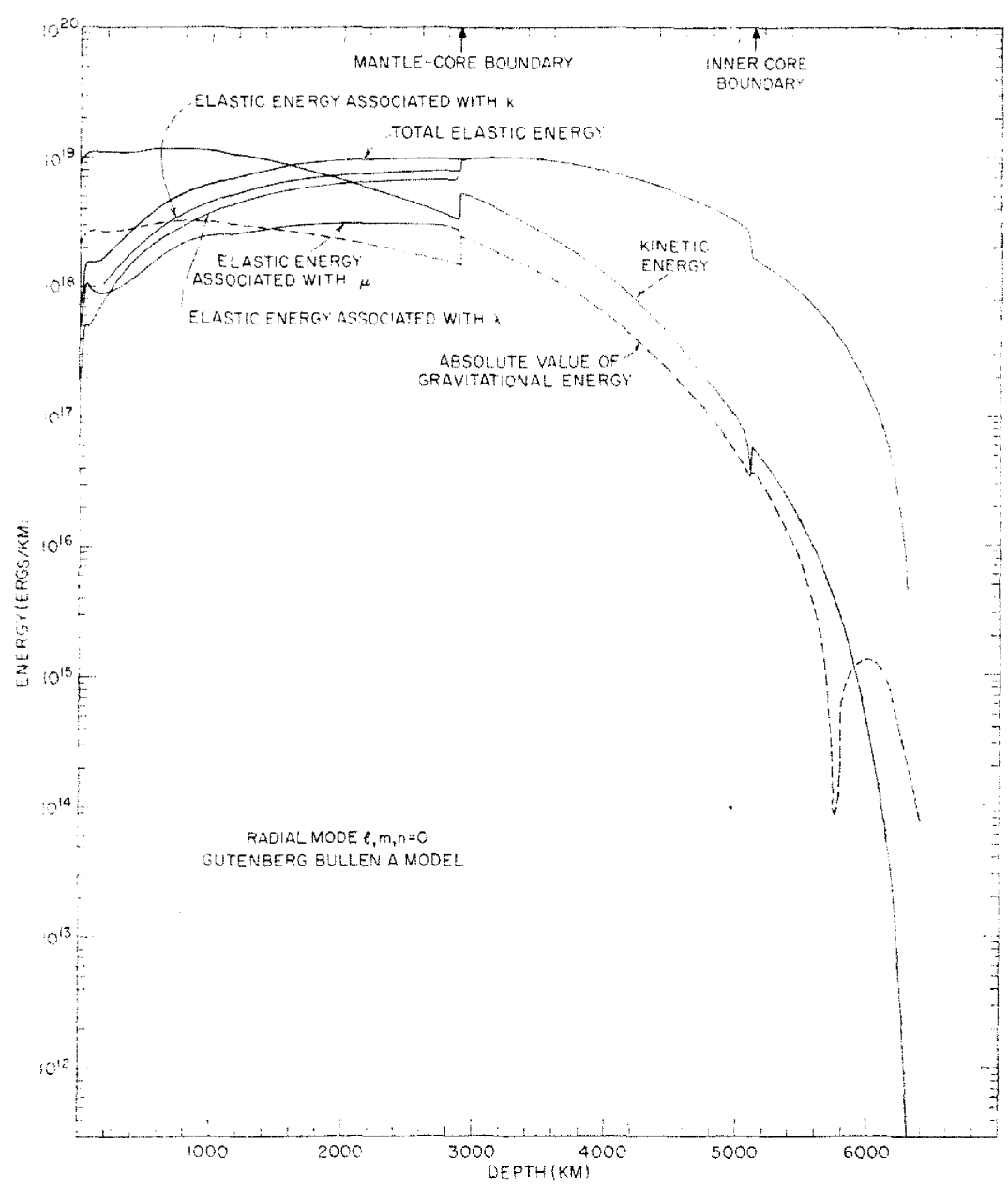

Fig. 3. Energy per unit radius for the radial mode. 


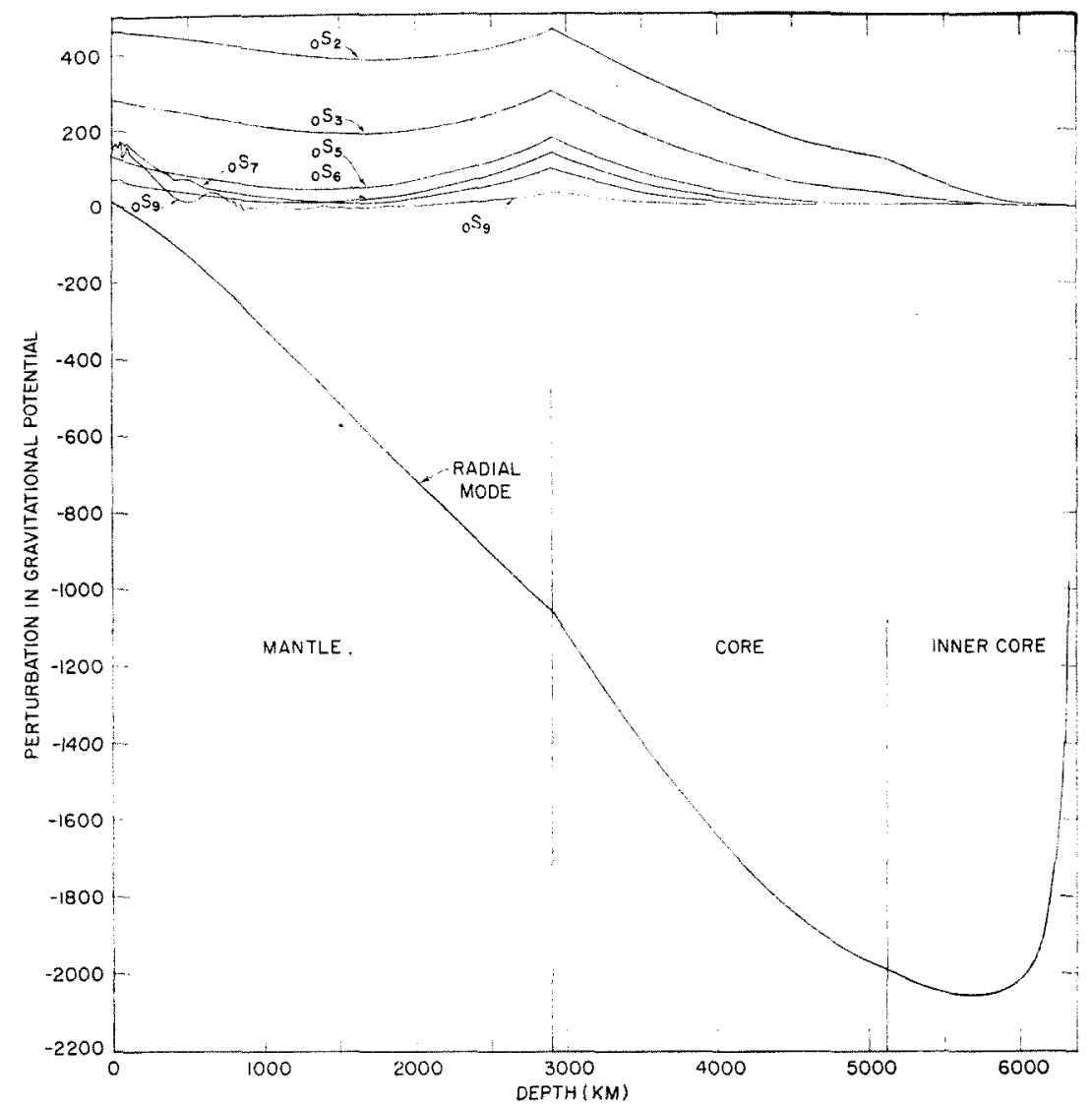

Fig. 4. Perturbation in the gravitational potential for the low-order spheroidal oscillations.

pression are much less than losses in shear, and since the upper mantle is more dissipative than the lower mantle, Figure 3 immediately explains the persistence of the $S_{0}$ mode compared with the other normal modes of the earth.

The gravitational energy per unit thick shell for $S_{0}$ closely resembles in shape that of the kinetic energy, except it is of opposite sign. The reversal in trend in the gravitational potential at a depth of about $5800 \mathrm{~km}$ is due to the fact that $d P / d r$ changes sign (Figure 4). For the radial mode the total kinetic energy averaged over a cycle is $0.28 \times 10^{23}$ ergs for a $1-\mathrm{cm}$ surface displacement. The total elastic energy is $0.37 \times 10^{22}$ ergs, whereas the gravitational energy is equal to $-0.09 \times 10^{23}$ ergs.

The variation with frequency of the total kinetic energy averaged over a cycle for the spheroidal oscillations is shown in Figure 5 and Table 4. The energy is normalized so that $m=$ 0 and the radial function in the vertical component of displacement ${ }_{n} U_{l}(r)$ is equal to $1 \mathrm{~cm}$ at the free surface. The kinetic energy averaged

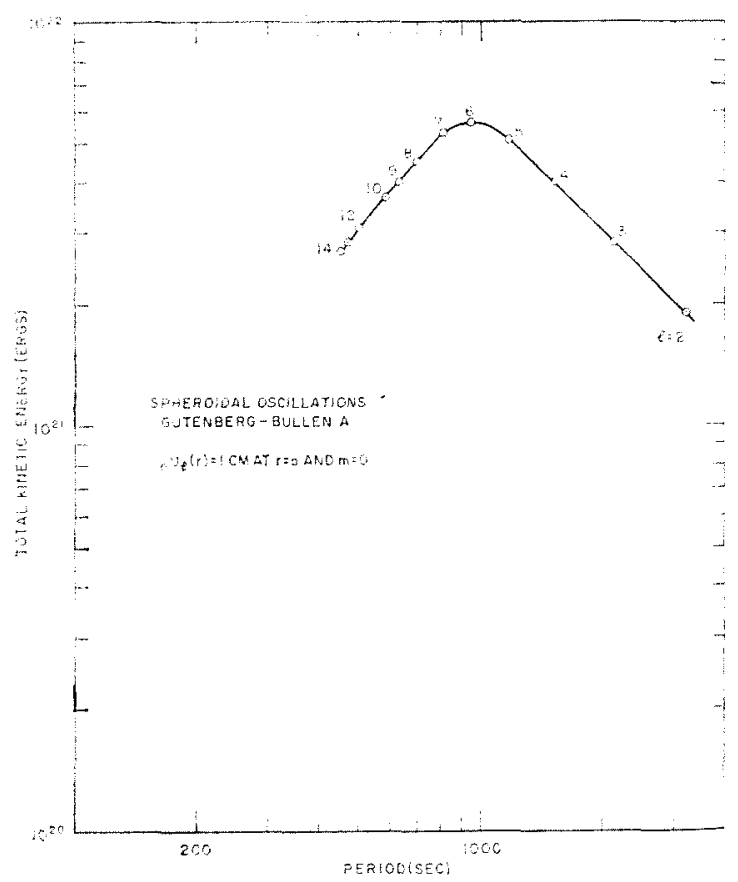

Fig. 5. Total kinetic cnergy versus period for fundamental mode spheroidal oscillations. 
TABLE 4. Energies for $0 S_{l}$

\begin{tabular}{cccc}
$\begin{array}{c}\text { Order, } \\
l\end{array}$ & $\begin{array}{c}T, \\
\text { sec }\end{array}$ & $m=0$ & $m=1$ \\
\hline & & \multicolumn{2}{c}{ Kinetic Energy, ergs } \\
2 & 3208 & $.189 \times 10^{22}$ & $.0113 \times 10^{24}$ \\
3 & 2120 & $.285 \times 10^{22}$ & $.0342 \times 10^{24}$ \\
4 & 1533 & $.406 \times 10^{22}$ & $.0812 \times 10^{24}$ \\
5 & 1180 & $.510 \times 10^{22}$ & $.1530 \times 10^{24}$ \\
6 & 955 & $.562 \times 10^{22}$ & $.2300 \times 10^{24}$ \\
7 & 807 & $.527 \times 10^{22}$ & $.2950 \times 10^{24}$ \\
8 & 703 & $.448 \times 10^{22}$ & $.3220 \times 10^{24}$ \\
9 & 634 & $.400 \times 10^{22}$ & $.3600 \times 10^{24}$ \\
10 & 579 & $.369 \times 10^{22}$ & $.3990 \times 10^{24}$ \\
12 & 504 & $.312 \times 10^{22}$ & $.4870 \times 10^{24}$ \\
13 & 473.0 & $.287 \times 10^{22}$ & $.5220 \times 10^{24}$ \\
14 & 448.1 & $.274 \times 10^{22}$ & $.5750 \times 10^{24}$ \\
15 & 426.5 & $.246 \times 10^{22}$ & $.5900 \times 10^{24}$ \\
& & &
\end{tabular}

over a cycle is equal to the sum of the elastic and gravitational energy. Spheroidal vibrations involve radial displacements and perturb the gravitational ficld by the wrinkling of interfaces and perturbation of the gravitational field. For $l>7$ the gravitational energy is neglible compared with the elastic energy.

In progressing from ${ }_{0} S_{2}$ to ${ }_{0} S_{a}$ the kinetic energy increases by about a factor of 3 to a maximum value at a period of about 940 sec. The shape of the kinetic energy curve is strikingly similar to the group velocity curve for very long-period Rayleigh waves [Oliver, 1962].

An insight into the behavior of spheroidal oscillations can be obtained by examining the radial distribution of the kinetic energy. Figures 6 and 7 illustrate the kinetic energy per unit radius for the Gutenberg model earth. For the

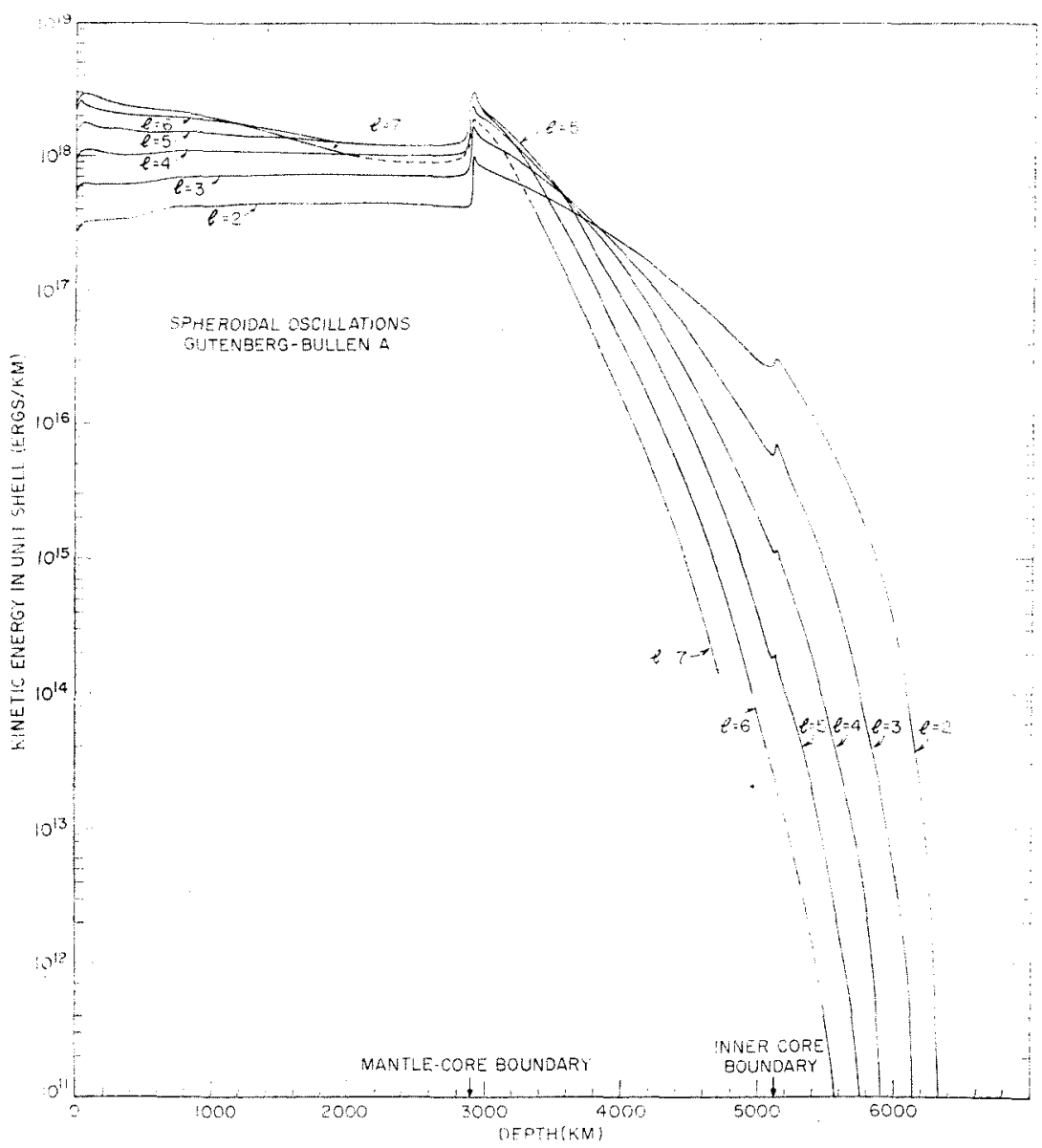

Fig. 6. Kinetic energy versus depth for spheroidal modes 2 through 7 for the GutenbergBullen A Model. 


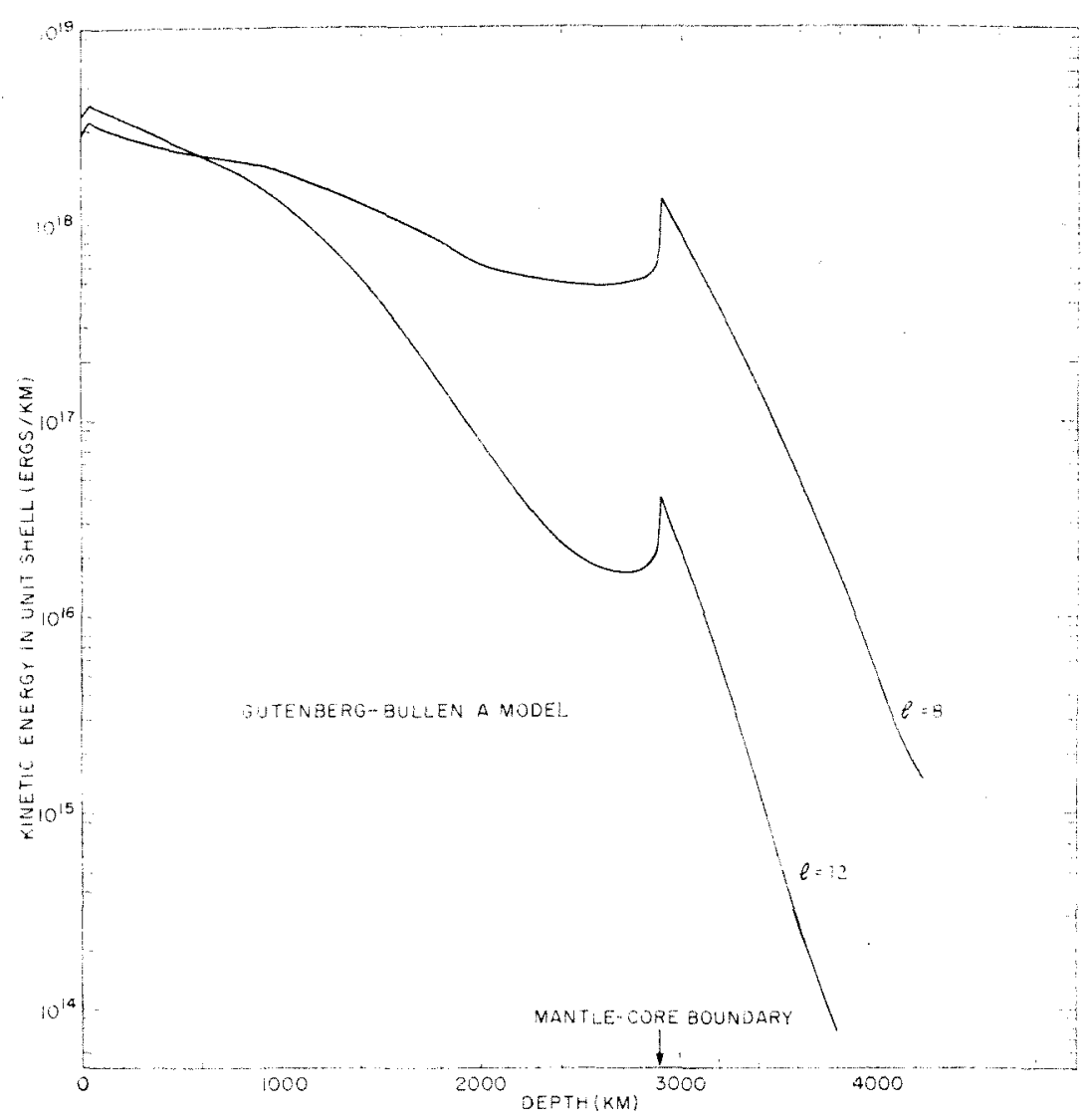

Fig. 7. Kinetic energy versus depth for spheroidal modes 8 and 12 .

low mode numbers the energy is fairly evenly distributed throughout the mantle and core. For $l>7$ the kinetic energy starts to concentrate toward the earth's surface. These curves show that the low-order spheroidal oscillations are particularly sensitive to the density of the outcr part of the core.

The kinetic encrgy is proportional to the local density times the squared sum of the vertical and horizontal displacements. Both these quantities are decreasing within the core. A slight reversal in the kinetic energy curve is present at a radius of $1250 \mathrm{~km}$. This is a result of the density jump at the boundary of the inner core.

The distribution of energy in the various parts of the earth for the various modes is of great importance in the interpretation of free oscillation data. The effect of a change in the elastic properties or density in a certain region of the earth on the period of a given mode is directly proportional to the appropriate fractional energy contained in that region for that mode [Archambeau and Anderson, 1964]. Inspection of Figure 6 reveals that only the first few spheroidal modes are sensitive to the density of the inner core. This information is only useful, however, to the extent that the density of the mantle and outer core are known. Looking at the kinetic energy variations, we sce that modes 2 through 7 all sample the whole mantle about equally and therefore are as sensitive to the density in the upper mantle as they are to the density in the lower mantle. A study of the low-order modes combined with knowledge of the mass and moment of inertia is greatly improving our knowledge of the gross density structure of the earth.

Figure 8 shows the variation of kinetic and potential energy per unit radius for the gravest spheroidal mode. Within the mantle the total elastic energy is slightly less than the kinetic energy to a depth of about $1800 \mathrm{~km}$. The total 
elastic energy then rises sharply approaching the core-mantle boundary.

The variation with depth of the $\lambda$ and $\mu$ components of the elastic potential energy are also shown in Figure 8. The elastic energy associated with $\lambda$ is much less than that associated with shear. In fact, beyond a depth of about $1800 \mathrm{~km}$, effectively all the elastic energy in the mantle is contributed by the rigidity component of the elastic energy. In contradistinction to the purely radial mode, Figure 3, the elastic energy for ${ }_{0} S_{2}$ is controlled almost exclusively by the rigidity properties of the mantle. This explains the higher damping of this mode relative to ${ }_{0} S_{0}$. The abrupt drop in the elastic energy by many orders of magnitude is a result of the rigidity dropping to zero within the fluid core. The elastic energy rises slightly approaching the inner core boundary. The perturbations in the elastic energy at depths of 4982 and $5121 \mathrm{~km}$ are a result of the discontinuities in compressional velocity and density, respectively (Table 1 ).

The elastic energy stored in the core is several orders of magnitude less than the kinetic energy. Since the elastic energy stored in the core is so small, it would be difficult to use free oscillation data to improve estimates of velocity in this region materially. On the other hand, the rapid rise of potential energy near the base of the mantle may permit improved estimates of the velocity to be made in this region, since this zone is difficult to study with body wave travel times. It is clear that this mode can be used to refine the density of the core.

Figure 9 shows the elastic energy per unit thick shell associated with the rigidity for the spheroidal modes 2 through 12 . For order numbers 2 through 5 the elastic energy increases toward the core boundary, demonstrating the primary influence of the properties of the lower mantle. For larger order numbers the elastic energy progressively concentrates at shallower depths. For example, the 500-sec period oscillation $(l=12)$ is dominated by the elastic properties at a depth of $1200 \mathrm{~km}$ within the mantle.

Energy ratios are also important in the study of the attenuation of free oscillations [Anderson and Archambeau, 1964]. Assuming that imperfections in elasticity are the main source of

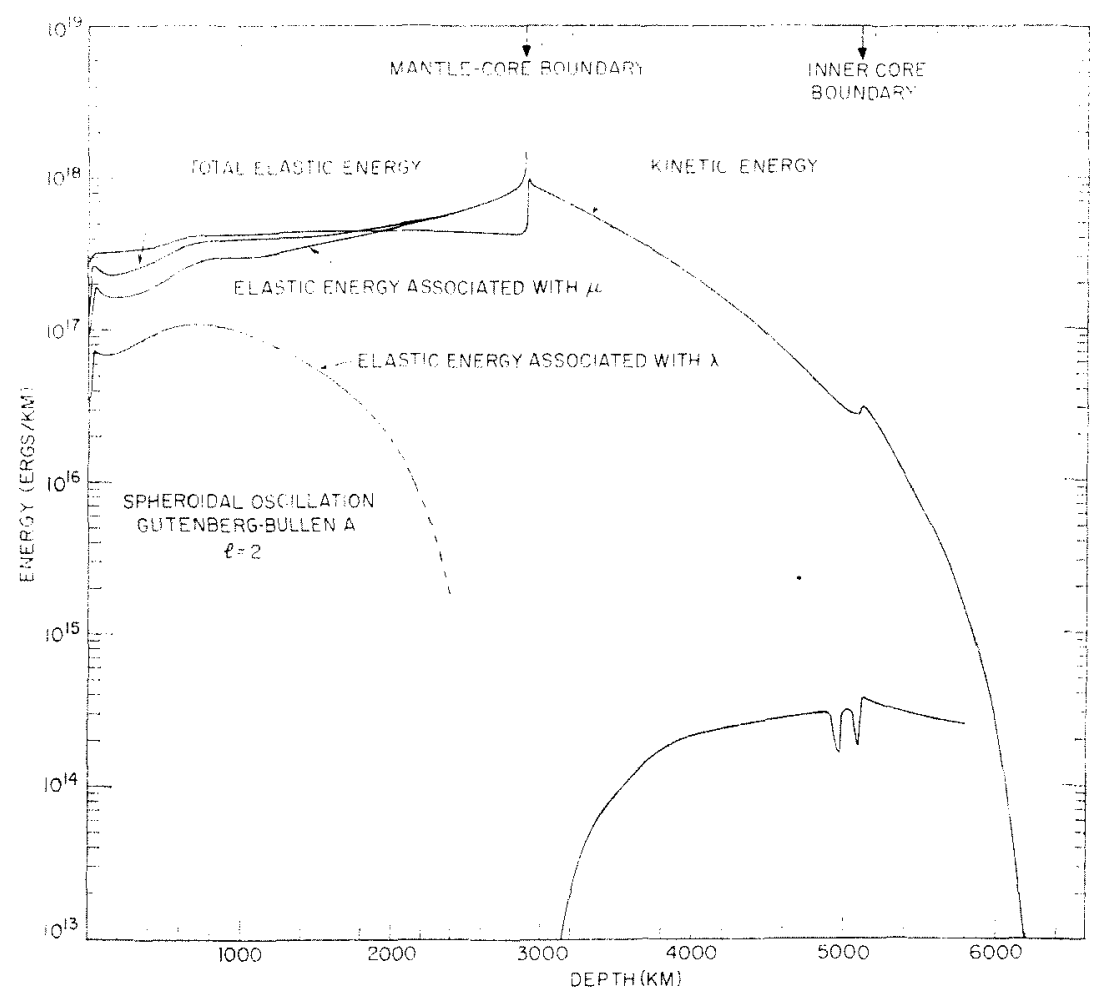

Fig. 8. Elastic and kinetic energy per unit radius for the gravest spheroidal mode ${ }_{0} S_{3}$. 


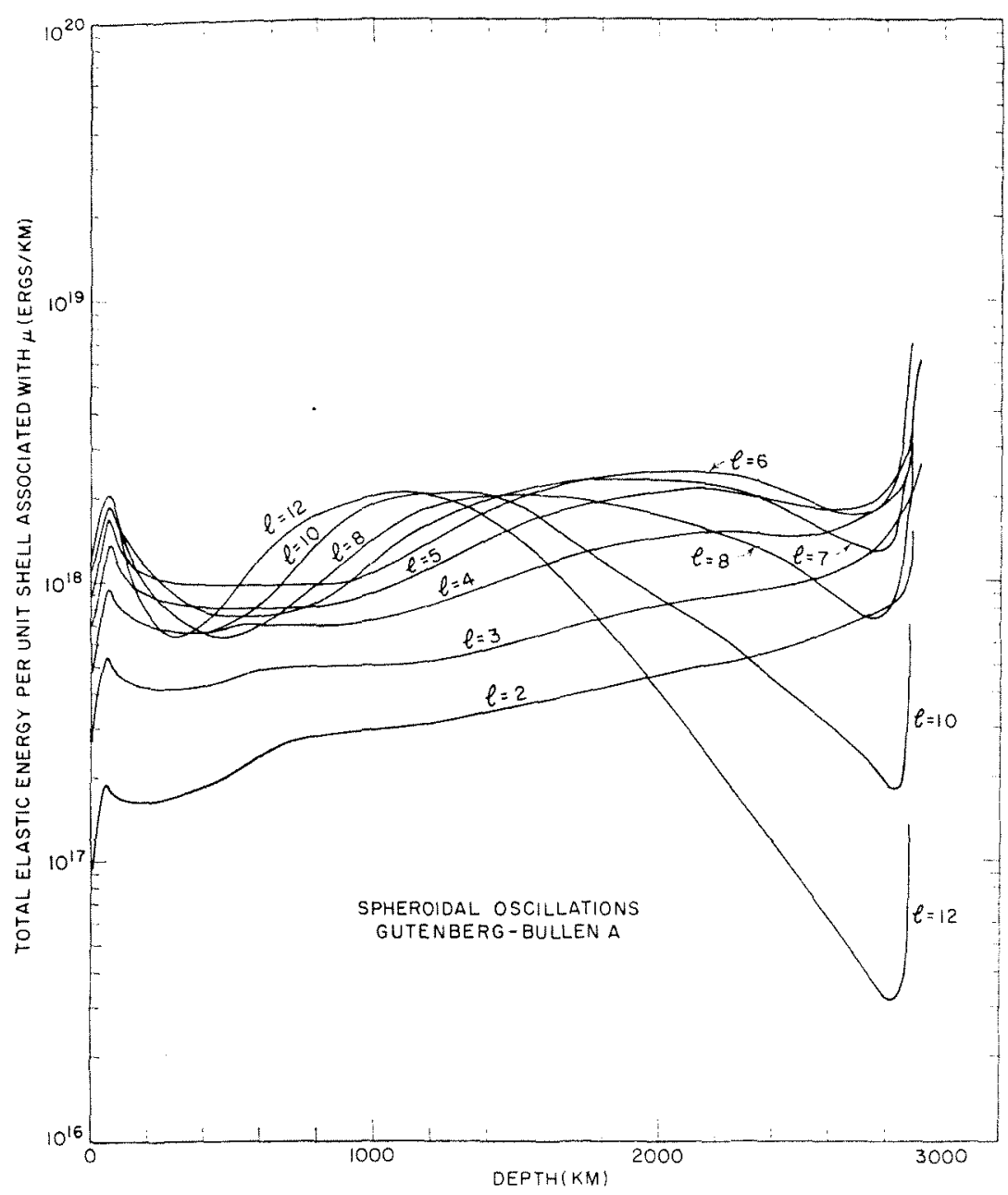

Fig. 9. Elastic energy per unit radius associated with rigidity for spheroidal modes 2 through 12 .

damping, it is clear that attenuation in the mantle probably dominates the observed decay of the free oscillations. We will treat this relation in detail in a future paper.

\section{Application to the Alaskan Earthquake}

The previous equations can be used to calculate the energy in several of the spheroidal oscillations excited by the Alaskan earthquake of March 28, 1964. Nowroozi [1965] presented observations of the mean amplitude of the vertical component of ground displacement recorded at Berkeley, California, for spheroidal modes ${ }_{0} S_{7}$ to ${ }_{0} S_{15}$ excited by the Alaskan shock (Table 5). The vertical amplitude is proportional to the tesseral harmonic $P_{l}{ }^{m}(\cos \theta) e^{i m \phi}$ where $\theta$ and $\phi$ are the appropriate angles be-
TABLE 5. Alaskan Earthquake March 28, 1964*

\begin{tabular}{lllll}
\hline Mode & $u, \mathrm{~cm} \dagger$ & ${ }_{0} U, \mathrm{~cm}$ & $\begin{array}{c}\text { Corrected } \\
\text { for } Q^{\dagger}\end{array}$ & $\begin{array}{c}\text { Energy, } \\
\text { ergs }\end{array}$ \\
\hline$S_{3}$ & .0303 & .00445 & .00430 & $6.30 \times 10^{21}$ \\
$0 S_{8}$ & .0283 & .00354 & .00390 & $9.70 \times 10^{21}$ \\
$0 S_{9}$ & .0220 & .00290 & .00387 & $1.21 \times 10^{22}$ \\
${ }_{0} S_{10}$ & .0092 & .00184 & .00250 & $1.14 \times 10^{22}$ \\
${ }_{0} S_{12}$ & .0056 & .00090 & .00153 & $1.13 \times 10^{22}$ \\
$0 S_{13}$ & .0136 & .00105 & .00225 & $2.10 \times 10^{22}$ \\
$0 S_{14}$ & .0097 & .00054 & .00110 & $1.28 \times 10^{22}$ \\
$0 S_{15}$ & .0147 & .000775 & .00177 & $2.30 \times 10^{22}$ \\
\hline
\end{tabular}

* Table taken from $W u$ [1966].

$\dagger$ Nowroozi [1965].

$\ddagger Q$ values taken from Alsop et al. [1961] and Benioff et al. [1961]. 
tween the source and receiver and not the geographical colatitude and longitude. The integer $m$ depends on the source mechanism.

For simplicity let us assume that $m=0$, and illustrate the kinetic energy computation for the ${ }_{0} S_{7}$ mode. The mean vertical surface displacement is $0.0303 \mathrm{~cm}$. Taking the angular distance between the Alaskan shock and Berkeley as $28.1^{\circ}$ allows the mean radial factor of displacement to be computed.

$\overline{{ }_{0} U_{7}(a)}=0.0303 / P_{7}{ }^{\circ}(\cos 28.1)=0.0747 \mathrm{~cm}$

We must next correct for the finite decay of the ${ }_{0} S_{7}$ oscillation. The amplitude at any time $t$ is given by the expression

$$
A(t)=A\left(t_{0}\right) \exp \left(-\pi\left\{t-t_{0}\right\} / Q T\right)
$$

where $T$ is the period of the wave, $Q$ is the dimensionless quality factor, and $t_{0}$ is the time of the first oscillation. Over any time interval $\tau$ an average estimate of the amplitude is made in any frequency analysis

$$
\bar{A}=\frac{1}{\tau} \int_{0}^{\tau} A(t) d t
$$

Therefore, the average amplitude can be written in terms of the initial amplitude and the sample length of the record analyzed as

$$
\bar{A}=\frac{-Q T}{\pi \tau}\left\{\exp \left(-\frac{\pi \tau}{Q T}\right)-1\right\} A\left(t_{0}\right)
$$

Taking ${ }_{0} Q_{7}$ as 370 and the analyzed record length of $731.5 \mathrm{~min}$ leads to a value of 0.0931 $\mathrm{cm}$ for the corrected initial radial factor of the surface displacement.

For $m=0$ only, the energy corresponding to the $S_{\text {- }}$ oscillation for the Alaskan earthquake is $0.0931 \times 0.527 \times 10^{22} \mathrm{ergs}$ or $4.9 \times 10^{20} \mathrm{ergs}$.

However, $m=0$ is not a realistic assumption for a shallow earthquake mechanism, since this assumption implies no azimuthal dependence at the source. If we assume that $m=2$ only, the vertical component of surface displacement would be divided by $P_{l}^{2}(\cos \theta) e^{2 i s}$ to determine the radial factor of the displacement. The calculation of energy then only involves multiplication by $(l+m) ! /(l-m)$ !

Since the distribution of energy in the various modes depends on the fault parameters at the source, it is probable that the observed energy for each order $l$ is a weighted average of $m=0,1$, and 2. In this case to determine $U_{l}(r)$ we must normalize by dividing the observed vertical component of displacement by

$$
\sum_{m=0}^{2} A_{l}{ }^{m} P_{l}^{m}(\cos \theta) e^{i m \phi}
$$

where the $A_{m}$ 's are weighting factors for each value of $m$ and where they are dependent on the source parameters and frequency. The observed energy in each order is then given by the weighted average

$$
\sum_{m=0}^{2}\left(A_{l}{ }^{m}\right)^{2} E_{m} U_{l}(r)
$$

where $E_{m}$ are the individual energy contributions from $m=0,1$, and 2 and $U_{l}(r)$ is the observed radial factor corrected for attenuation losses.

$W u$ [1966] has studied the observed free oscillation energy, using appropriate weighting factors for the source mechanism of the Alaskan earthquake and the theoretical results presented herein. Some of these results are summarized in Table 5 .

A total energy of the order of $10^{23}$ ergs is indicated in the spheroidal modes for the Alaskan shock over the period range from 450 to $850 \mathrm{sec}$. From the published magnitude of 8.4 a total energy release of $3.3 \times 10^{24} \mathrm{ergs}$ is obtained from the relation

$$
\log E=11.8+1.5 M
$$

This relationship would imply that the free oscillation energy density must increase toward high frequencies if the total energy in the earthquake is of the order of $10^{24}$ to $10^{25} \mathrm{ergs}$. The body wave determination of magnitude for the Alaskan shock can also be questioned; it is probably an underestimate. Press and Jackson [1965], using. static deformation results, estimate $10^{2-}$ ergs as an upper limit for the energy release.

The energy contained in the aftershocks that occurred in the time window of the analysis will raise the measured energy - the values in Table 5 are therefore upper limits for the energy of the main shock. For example, there were 26 reported aftershocks of magnitude greater than 4.7 in the time window covered by Nowroozi's analysis; 9 of these aftershocks had magnitude of 6.2 or greater. 
Acknowledgments. This research was partially supported by the Advanced Research Projects Agency and was monitored by the Air Force Office of Scientific Research under contracts AF 49(638)-1687 (SU) and AF 49(638)-1337 (CIT).

\section{REFERENCES}

Alsop, L. E., G. H. Sutton and M. Ewing, Measurement of $Q$ for very long period free oscillations, J. Geophys. Res., 66, 2911-2916, 1961.

Alterman, Z., H. Jarosch, and C. L. Pekeris, Oscillations of the earth, Proc. Roy, Soc. London, A, $252,80-95,1959$.

Anderson, Don L., Universal dispersion tables, 1, Love waves across oceans and continents on a spherical earth, Bull. Seismol. Soc. Am., 54, 681$726,1964$.

Anderson, Don L., and C. B. Archambeau, The anelasticity of the earth, J. Geophys. Res., 69. 2071-2084, 1964.

Archambeau, C. B., and Don L. Anderson, Inversion of surface wave dispersion data, $P u b-$ lication du Bureau Central Seismologique International, Série $A$, Travaux Scientifiques. Fascicula 23, 45-54, 1964.

Benioff, H., F. Press, and S. Smith, Excitation of the free oscillations of the earth by earthquakes. J. Geophys. Res., 66, 605-615, 1961.

Harkrider, D. G., Surface waves in multilayered elastic media, 2, Higher mode spectra and spectral ratios from point sources in plane layered earth models, Bull. Seismol. Soc. Am., in press, 1966.

Harkrider, David G., and Don L. Anderson, Surface wave energy from point sources in plane layered earth models, J. Geophys. Res., $\pi 1$, 2967-2980, 1966.

Haskell, N. A., Total energy and energy spectral density of elastic wave radiation from propagating faults, 2, A statistical source model, Bull. Seismol. Soc. Am., 56, 125-140, 1966.

Hoskins, L. M., The strain of a gravitating sphere of variable density and elasticity, Trans. Am. Math. Soc., Q 1, 1-43, 1920.
Jeffreys, H., The Pamir earthquake of 18 February 1911 in relation to the depths of earthquake foci, Monthly Notices Roy. Astron. Soc., Geophys. Suppl., 1, 22-31, 1923.

Jobert, G., and N. Jobert, Application du principe de Rayleigh a la dispersion d'ondes superficielles, Ann. Geophys., 9, 250-255, 1953.

Love, A. E. H., A Treatise on the Mathematical Theory of Elasticity, Cambridge University Press, New York, 1927.

MacDonald, Gordon J. F., and Norman F. Ness, A study of the free oscillations of the earth, $J$. Geophys. Res., 66, 1865-1911, 1961.

Morse, P. M., and H. Feshbach, Methods of Theoretical Physics, McGraw-Hill Book Co., New York, 1953.

Nowroozi, Ali A., Eigenvibrations of the earth after the Alaskan earthquake, $J$. Geophys. Res., $70,5145-5156,1965$.

Oliver, J., A summary of observed seismic surface wave dispersion, Bull. Seismol. Soc. Am., 52, 81-86, 1962.

Pekeris, C. L., and H. Jarosch, The free oscillations of the earth, in Contributions in Geophysics in Honor of Beno Gutenberg, Pergamon Press, New York, 1958.

Press, F., and W. Jackson, Alaskan earthquake, 27 March 1964: Vertical extent of faulting and elastic strain energy release, Science, $147,867-$ $869,1965$.

Stoneley, R., The variation of amplitude and energy with depth in Love waves, in Contributions in Geophysics in Honor of Beno Gutenberg, pp. 36-43, Pergamon Press, New York, 1958.

Takeuchi, H., J. Dorman, and M. Saito, Partial derivatives of surface waves phase velocity with respect to physical parameter changes within the earth, J. Geophys. Res., 69, 3429-3441, 1964.

Wu, Franeis T., Lower limit of the total energy of earthquakes and partitioning of energy among seismic waves, Ph.D. Thesis, California Institute of Technology, 1966.

(Received September 16, 1966.) 